\title{
Does familiarity with CDC guidelines, continuing education, and provider characteristics influence adherence to chronic pain management practices and opioid prescribing?
}

Jean C. McCalmont, DNP, FNP; Kim D. Jones, PhD, FNP, FAAN; Robert M. Bennett, MD, FRCP, MACR; Ronald Friend, PhD

\section{ARTICLE INFO}

\section{Keywords:}

chronic pain

2016 CDC guideline

opioid prescribing

provider practice
DOI:10.5055/jom.2018.0437

(C) 2018 Journal of Opioid Management, All Rights Reserved.

\begin{abstract}
Objectives: (1) To assess providers' experience and knowledge of chronic noncancerpain (CNCP) management. (2) To assess providers' utilization of the Centers for Disease Control and Prevention (CDC) 2016 Guideline for Prescribing Opioids for Chronic Pain. (3) To assess the influence of the 2016 CDC guideline on provider confidence in managing CNCP and adherence to the CDC recommendations.

Methods: A cross-sectional, web-based survey conducted with 417 Oregon prescribing providers, divided into three continuing medical education (CME) groups composed of minimal ( $0-3)$, moderate (4-10), and high ( $\geq 11$ ) hours of training.

Results: The three CME groups were associated with increased use of CDC opioid recommended practices (29.4, 34.2, 38.8; $p=0.001$; scale 0-50), opioid conversion confidence (5.5, 6.5, 7.4; $p<0.001$; scale 0-9), and confidence in pain management (5.5, 5.9, 6.9; $p<0.001$, scale 0-9). Slightly more providers utilized CDC recommended practices than did not ( 57 vs 43 percent). However, CME groups differed substantially in utilization of CDC practices (42 vs 57 vs 72 percent; $p<0.001$ ). Neither providers' profession (physician vs nurse practitioner [NP]) nor geographic setting (urban vs rural) showed differences in use of recommended practices or general confident in pain management (all p>0.05); however, physicians were slightly more confident in opioid dose conversion than NPS (6.9 vs 5.9; $p<0$. 001, scale 0-9).
\end{abstract}

Conclusions: Higher hours of recent CME positively benefit provider confidence in pain management and utilization of CDC recommended practices. NPs and rural providers were equivalent to their physician and urban counterparts on confidence and adherence to CDC practices, with minor exceptions.

\section{INTRODUCTION}

Chronic pain is a common complaint in the primary care setting. Some 33-45 percent of primary care patients report having chronic pain. ${ }^{1,2}$ Given the limited number of pain specialists, primary care providers deliver the majority of pain care in the United States. Despite this, most primary care providers have little formal training in pain management and opioid prescribing practices. ${ }^{3}$ In fact, a 2011 evaluation of US and Canadian medical schools ${ }^{4}$ found that the cumulative number of pain teaching hours for participating medical schools in the United States ranged from 1 to 31, with a mean of 11.13 $( \pm 8.23)$ hours. Many primary care providers have inadequate knowledge of chronic pain management practices ${ }^{5-7}$ with a resulting lack confidence in caring for chronic noncancer pain (CNCP) patients; not surprisingly, these providers consistently report high levels of frustration. 8,9

In an effort to promote safe prescribing practices among primary care providers and reduce the rate 
of adverse events related to opioid use, the CDC released its Guideline for Prescribing Opioids for Chronic Pain in March 2016. ${ }^{10}$ While the release of this guideline may be an important first step, they are not without controversy. ${ }^{11}$ Further, a systemic review found that clinical practice guidelines have little or no effect on promoting behavioral change among healthcare professionals. ${ }^{12,13}$ Surveys of medical students and physicians have found that they prioritize patient information as well as personal experience and intuition when making chronic pain treatment decisions, rather than drawing on evidence-based guidelines. ${ }^{14,15}$ There is concern that these recommendations are not user friendly, being based mainly on safety rather than efficacy, and not sufficiently reflecting the patient or provider needs. ${ }^{16}$

Herein, we evaluated Oregon healthcare providers' training, utilization of the CDC guideline and their perception of this guideline's opioid threshold dose ( $\leq 90$ morphine milligram equivalents [MME]/ d). We also examined contextual factors, which may influence a provider's confidence and adherence to current opioid prescribing recommendations, such as continuing medical education (CME), professional training, and geographic location.

\section{METHODS}

\section{Questionnaire and measures}

We conducted an anonymous, cross-sectional, investigator designed, 31-item survey (see Appendix 1) between January and April 2017 using a convenience sample of Oregon healthcare providers. Survey refinement was completed in conjunction with key stakeholders including pain management experts and healthcare providers who directly contributed to the CDC guideline. Involving key stakeholders in the development and revision of the survey tool also helped to mitigate potential nonresponse bias.

Survey questions were derived from prominent themes in the literature and central tenets of the CDC guideline. Measures fall into five broad categories:

a. Familiarity with CDC guideline: Familiarity with CDC guideline was assessed by selection of one of the following alternatives (a) not familiar with guidelines, (b) familiar but have not read, (c) have read, but not applied in practice, and (d) have read and applied in practice. A second question assessed opinion on the CDC's recommendation that clinicians avoid prescribing opioid doses $\geq 90 \mathrm{MME} / \mathrm{d}$. Respondents were asked if this threshold dose was (a) too high, (b) reasonable, or (c) too low. These questions were modified from Morse et al. ${ }^{17}$ A synopsis of the CDC guideline is included in Appendix 2.

b. Provider confidence in managing chronic pain and calculating opioid conversion dose (see Table 2): "How confident are you in managing chronic noncancer pain?" (management confidence), and "How confident are you calculating opioid conversion doses (morphine equivalents) of commonly used opioids?" ("conversion confidence"; $0=$ not confident, $9=$ very confident). The correlation between the two confidence measures was $r=0.48$.

c. Provider concern surrounding long-term opioid use in CNCP patients (see Table 2): "How concerned are you that your patients on long-term opioids will ....(a) Develop psychological dependence?; (b) Develop physical dependence (experience withdrawal symptoms if medication is tapered too quickly or stopped abruptly)?; (c) Develop opioid use disorder (addiction)?; (d) Divert their opioids?; (e) Overdose?; and, (f) Develop opioid-induced hyperalgesia?" ( 0 = not concerned, 9 = very concerned).

\section{d. Provider utilization of the CDC recommen-} dations: Ten recommended annual practices $(0=$ never, $5=$ always $)$ for providers prescribing to patients starting on opioids were summed to form a "Composite Score of CDC Opioid Management Recommendations" referred now as "CDC Composite Score" (0-50). Those 10 items were: selection of nonpharmacologic therapy and nonopioid medications instead of opioids; screening for comorbid mental health disorder; use of an opioid risk assessment tool (eg, opioid risk tool); review of a patient's personal or family history of substance abuse; requiring an opioid treatment agreement; establishing treatment goals with the patient; conducting a urine drug screening; 
accessing the Oregon prescription drug monitoring program (PDMP); referring patient to a pain management specialist; and assessing for benzodiazepine use. Four of these measures (use of an opioid risk tool, urine screening, PDMP, and opioid treatment agreement) were analyzed individually because of their significance. The 10 items comprising the Composite Score have a mean of 34.0 ( $\mathrm{SD}=11$; range $0-50)$, Cronbach's $\alpha=0.90$, and average inter item correlation of 0.49 .

e. CME training in CNCP management in the past 2 years: Three CME groups were created based on natural breaks in the data and the Oregon state one-time requirement that prescribing providers complete 6 hours of CME in pain management, end of life care, or a combination of both. ${ }^{18}$ The three CME groups are: 130 individuals with minimal recent CME (0-3h), 168 individuals with moderate recent CME (4-10 h), and 119 individuals with high recent CME (>11 h).

\section{Participants}

Inclusion criteria included: licensure as a prescribing healthcare provider (physician, NP, physician assistant, or medical resident). Exclusion criteria included providers who do not manage CNCP, nonprescribing providers (eg, registered nurses), and students. Although the target population for this survey was Oregon primary care providers, specialty providers were included in the analysis for comparison purposes. No financial compensation for participation was provided. The Oregon Health \& Science University Institutional Review Board approved this study, and informed consent was obtained from all survey respondents.

This survey was e-mailed to both individual providers as well as organizational leaders (eg, clinic managers, medical directors, directors of regional pain/opioid taskforces), who forwarded the survey link and consent information form link on to their provider staff. Jean C. McCalmont sent a total of 5,468 e-mails, 2,407 of which were sent to members of the Oregon State Board of Nursing list serve, 3,026 of which were sent to members of the Oregon Medical Board, and 35 of which were sent to organizational leaders. The organizational leaders forwarded the survey link and consent information form link on to approximately 2,211 individual providers. Based on these estimates, 7,679 prescribing providers in Oregon potentially received the survey link.

\section{Statistical analysis}

Analysis of variance and multivariate analysis of variance were used to analyze combinations of independent and dependent variables and their interactions. A primary focus was on the effect of the three levels of CME hours (minimal, moderate, and high) and provider characteristics (profession and geographic region) on opioid practices and confidence in pain management and opioid dose conversion. Multiple regression was also used to assess the amount of variance explained by multiple predictors. Mean substitution was used to address missing data where this occurred. $\chi^{2}$ were used to assess relations between categorical variables. Survey questions assessed on a 1-10 scale were converted to 0-9 scores for statistical purposes. Analyses were conducted by use of Statistica 13.2. Alpha levels and confidence intervals of 0.05 were applied.

\section{RESULTS}

Seven hundred twenty-three individuals accessed the survey (12 percent response rate). One hundred thirty-eight survey responses were excluded after applying the exclusion criteria. Notably 135 (18.6 percent) of the respondents did not manage chronic pain patients. After excluding 285 individuals who did not indicate their profession, there were a total of 438 survey responses. Twenty one physician assistants were excluded because of their small, and by comparison, unequal sample size.

A total of 417 participants remained and were analyzed statistically. Participants were equally divided between NPs $(n=217)$ and physicians $(n=210)$ and had an average of 15.6 years of practice. Two thirds of the sample was primary care providers and 66 percent of respondents were female prescribers. Seventeen of the respondents were specialists in pain medicine; their responses were examined to check for the questionnaire's validity. Demographic and provider characteristics are shown in Table 1.

Ninety five percent of the providers have started patients on opioids, and 64 percent of providers have prescribed opioids chronically ( $>3$ months). Providers' mean confidence in CNCP management 


\begin{tabular}{|l|l|}
\hline \multicolumn{2}{|c|}{ Table 1. Participant demographics } \\
and characteristics*
\end{tabular}

was 5.95 (0-9) and their mean confidence in opioid dose conversion was 6.4 (0-9). With regards to concern for patients on long term opioids, providers were most concerned about psychological dependence and physical dependence and least concerned about opioid diversion and overdose (see Table 2).

\section{CDC familiarity, threshold dose, provider characteristics, and CME}

To assess usefulness of the CDC guideline, providers were asked about their familiarity and utilization of the CDC guideline (Table 3). Fifty seven percent of respondents reported that they had read the CDC guideline and applied it in practice. Twelve percent of respondents had read the guideline, but had not applied it in practice, 22 percent were familiar with the guideline, but had not read it, and 18 percent had no familiarity with this guideline. Only

\section{Table 2. Percent concern and confidence in $\mathrm{CNCP}$ pain management and opioid prescribing by providers, ranked from highest to lowest $(\mathrm{N}=417) *$}

"How concerned are you that your patients on long-term opioids will ..."

\begin{tabular}{|l|c|c|c|}
\hline & $\begin{array}{c}\text { Low } \\
\text { concern } \\
\text { (0-3), } \\
\text { percent }\end{array}$ & $\begin{array}{c}\text { Moderate } \\
\text { concern } \\
\text { (4-6), } \\
\text { percent }\end{array}$ & $\begin{array}{c}\text { High } \\
\text { concern } \\
\text { (7-9), } \\
\text { percent }\end{array}$ \\
\hline $\begin{array}{l}\text { "Develop } \\
\text { psychological } \\
\text { dependence" }\end{array}$ & 6.0 & 18.5 & 75.5 \\
\hline $\begin{array}{l}\text { "Develop physical } \\
\text { dependence" }\end{array}$ & 5.0 & 20.8 & 74.1 \\
\hline $\begin{array}{l}\text { "Develop opioid } \\
\text { use disorder" }\end{array}$ & 13.7 & 26.4 & 59.9 \\
\hline $\begin{array}{l}\text { "Develop } \\
\text { opioid-induced } \\
\text { hyperalgesia" }\end{array}$ & 10.6 & 30.5 & 58.9 \\
\hline "Overdose" & 28.5 & 35.3 & 36.2 \\
\hline $\begin{array}{l}\text { "Divert their } \\
\text { opioids" }\end{array}$ & 26.6 & 38.1 & 35.3 \\
\hline "How & & & \\
\hline
\end{tabular}

\begin{tabular}{|l|c|c|c|}
\hline "How confident are you in ..." & $\begin{array}{c}\text { Low } \\
\text { confidence } \\
\mathbf{( 0 - 3 ) ,} \\
\text { percent }\end{array}$ & $\begin{array}{c}\text { Moderate } \\
\text { confidence } \\
\mathbf{( 4 - 6 ) ,} \\
\text { percent }\end{array}$ & $\begin{array}{c}\text { High } \\
\text { confidence } \\
\mathbf{( 7 - 9 ) ,} \\
\text { percent }\end{array}$ \\
\hline $\begin{array}{l}\text { "Calculating opioid } \\
\text { conversion doses } \\
\text { (morphine equiva- } \\
\text { lents) of commonly } \\
\text { used opioids" }\end{array}$ & 16.5 & 23.3 & 60.2 \\
\hline $\begin{array}{l}\text { "Managing chronic } \\
\text { noncancer pain" }\end{array}$ & 11.0 & \multicolumn{1}{|c|}{45.2} & 43.8 \\
\hline $\begin{array}{l}\text { "Seventy five percent of providers are concerned with psy- } \\
\text { chological and physical dependence, and slightly more than } \\
\text { a third are concerned with overdose and diversion. Providers } \\
\text { are somewhat more confident in opioid dose conversion than } \\
\text { in pain management. }\end{array}$ & \\
\hline
\end{tabular}

slightly more providers (57 percent) applied the CDC guideline (ie, "read and applied") than those who responded to the other categories combined (43 percent), who had not applied the guidelines (Table 3). Recent CME training was associated with a 30 percent increase in use of the CDC guideline (42, 57, 72 percent; $p<0.001)$. There was no significant difference between physicians and NPs $(p>0.984)$ or between providers in urban and rural 
Table 3. Participants responses (percent) to CDC familiarity categories for total sample, CME training, profession, and region*

\begin{tabular}{|c|c|c|c|c|c|c|c|c|}
\hline $\begin{array}{c}\text { CDC } \\
\text { familiarity } \\
\text { categories }\end{array}$ & $\begin{array}{c}\text { Total, } \\
\mathbf{n}=\mathbf{4 1 7}\end{array}$ & $\begin{array}{c}\mathrm{CME}^{\dagger} \\
(\mathbf{0 - 3 \mathbf { h }}) \\
\mathbf{n}=\mathbf{1 3 0}\end{array}$ & $\begin{array}{c}\mathrm{CME}^{\dagger} \\
(4-10 \mathrm{~h}), \\
\mathbf{n}=168\end{array}$ & $\begin{array}{c}\mathrm{CME}^{\dagger} \\
(\geq 11 \mathrm{~h}), \\
\mathbf{n}=119\end{array}$ & $\begin{array}{c}\text { PHY } \\
\text { n }=210\end{array}$ & $\begin{array}{c}\text { NP } \\
\mathbf{n}=\mathbf{2 0 7}\end{array}$ & $\begin{array}{l}\text { Urban }^{\S} \\
\mathbf{n}=\mathbf{2 6 7}\end{array}$ & $\begin{array}{l}\text { Rural }^{\S}, \\
\text { n }=147\end{array}$ \\
\hline Not familiar & 9.6 & 16.9 & 8.3 & 3.4 & 9.0 & 10.1 & 12.0 & 5.4 \\
\hline $\begin{array}{l}\text { Familiar not } \\
\text { read }\end{array}$ & 22.3 & 32.3 & 22.0 & 11.8 & 22.4 & 22.2 & 22.8 & 21.1 \\
\hline $\begin{array}{l}\text { Read not } \\
\text { applied }\end{array}$ & 11.5 & 8.5 & 13.1 & 12.6 & 11.4 & 11.6 & 12.4 & 10.2 \\
\hline $\begin{array}{l}\text { Read and } \\
\text { applied }\end{array}$ & 56.6 & 42.3 & 56.5 & 72.3 & 57.1 & 56.0 & 52.8 & 63.3 \\
\hline
\end{tabular}

"Column 2 presents the percentage of the total sample choosing the four CDC familiarity categories: only 57 percent report having "applied" the CDC guidelines. The three CME groups in columns 3, 4, and 5 show a strong significant difference in their choices of the four CDC Categories. Forty nine percent with "minimal CME" hours (16.9+32.3 percent) are not familiar or have not read the CDC guidelines. The three CME training groups differ significantly in the "read and applied" category ( 42.3 vs 56.5 vs 72.3 percent). Columns 6 and 7 show identical responses in all four categories for NPs and Physicians ( $p<0.984)$. Columns 8 and 9 show a marginal significance $(\mathrm{p}<0.088)$. In comparing rural and urban providers, slightly more rural providers chose "read and applied" (63.3 vs 52.8 percent) and slightly more urban providers chose "not familiar" (12.5 vs 5.4 percent) with the CDC guidelines.

${ }^{\dagger} \mathrm{CME}: \chi^{2}(\mathrm{df}=6)=35.54 ; \mathrm{p}<0.001$.

${ }^{\prime} \mathrm{PHY}$ versus NP: $\chi^{2}(\mathrm{df}=3)=0.16 ; \mathrm{p}<0.984$.

${ }^{8}$ Urban versus Rural: $\chi^{2}(\mathrm{df}=3)=6.55 ; \mathrm{p}<0.088$.

settings $(p<0.088)$ in their choices of the four CDC familiarity categories (Table 3).

Providers were asked about their perception of the guideline's recommendation for the maximum MME dose of $\leq 90 \mathrm{MME} / \mathrm{d}$ ). Overall primary care providers thought that the dose was reasonable or too high (66.6 percent, reasonable; 26.6 percent, too high; 6.7 percent, too low). The 17 pain specialists were evenly divided in their responses (too low, 35 percent; reasonable, 35 percent; too high, 29 percent). Notably, while 35 percent of pain specialists said they were too low, less than 10 percent of nonpain specialist NPs and physicians said they were too low. There was no difference between physicians and NPs $(\mathrm{p}<0.083)$ nor urban and rural providers $(\mathrm{p}<0.310)$ in threshold dose assessment.

\section{CME is associated with adherence to CDC practices and increased provider confidence}

The responses to annual use of 10 opioid practices $(0=$ never; $5=$ always $)$ were summed to provide a score ranging from 0 to 50 to form the "Composite Score of CDC Opioid Management Recommendations." As Figure 1A shows, the means for the three CME groups, minimal (29. 4, SD = 13.2), moderate $(34.2, \mathrm{SD}=11.2)$, and high $(38.8, \mathrm{SD}=7.8)$, were significantly different for increased practices with CME $(<0.001$; scale 0-50). Four of the specific practices (development of an opioid treatment agreement, use of the Oregon PDMP, use of urine drug screening, and application of an opioid risk tool) are shown in Figure 1B. All three CME groups differed significantly in their adherence to these four practices $(\mathrm{p}<0.001)$. Utilization of CDC practices in developing treatment plans did not differ appreciably from those actually used (3.4 vs 3.3, scale 0-5). Greater CME hours were associated with significantly increased opioid dose conversion confidence (5.5, $6.5,7.4 ; \mathrm{p}<0.001$; scale $0-9)$ and confidence in pain management $(5.5,5.9,6.9 ; \mathrm{p}<0.001$, scale $0-9)$ as shown in Figure 1C. A multiple $\mathrm{R}=0.51$ showed that CME, familiarity with the CDC guideline, confidence in pain management, and confidence in opioid dose conversion were significant and independent predictors (all $\mathrm{p}<0.001$ ) of the Composite Score of CDC Opioid Management recommendations; explaining approximately equal amounts of the 25 percent variance. When included in the equation, neither professional status $(\mathrm{p}<0.358)$ nor region $(\mathrm{p}<0.495)$ was a significant predictor. Some construct validity for a main portion of the questionnaire is supported by the fact that four primary study variables (management confidence, conversion confidence, CDC 

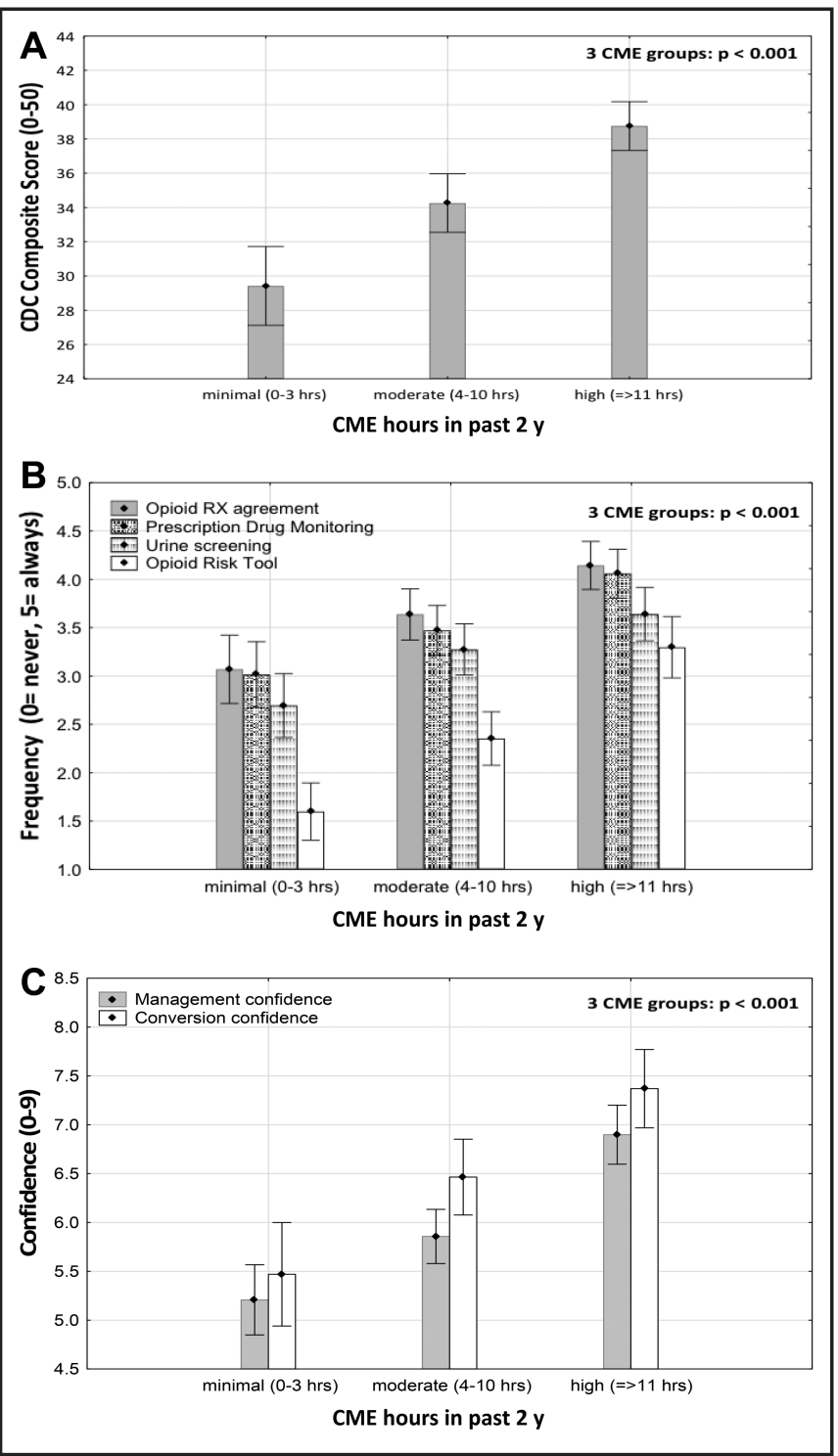

Figure 1. A, The three CME groups are significantly different from each other in the CDC Composite Score composed of the sum of 10 questions on opioid management $(F=22.12 ; p<0.001)$. $B$, The three $C M E$ groups are significantly different $(F=7.59 ; p<0.001)$ overall, and on each of the four specific CDC opioid management tools (all $p<0.001$ ), with the largest difference for the "opioid risk tool" $(\mathrm{F}=28.87 ; \mathrm{p}<0.001)$. C, The three CME groups are significantly different from each other for both Management Confidence $(F=26.01 ; p<0.001)$ and Conversion Confidence $(F=16.29 ; p<0.001)$.

familiarity, and CME training) uniquely predicted the 10 CDC adherence practices (Composite Score).

\section{Physician and NP comparisons with opioid} practices and confidence ratings

Figures 2A-2C show no significant differences between physician and NP providers in the
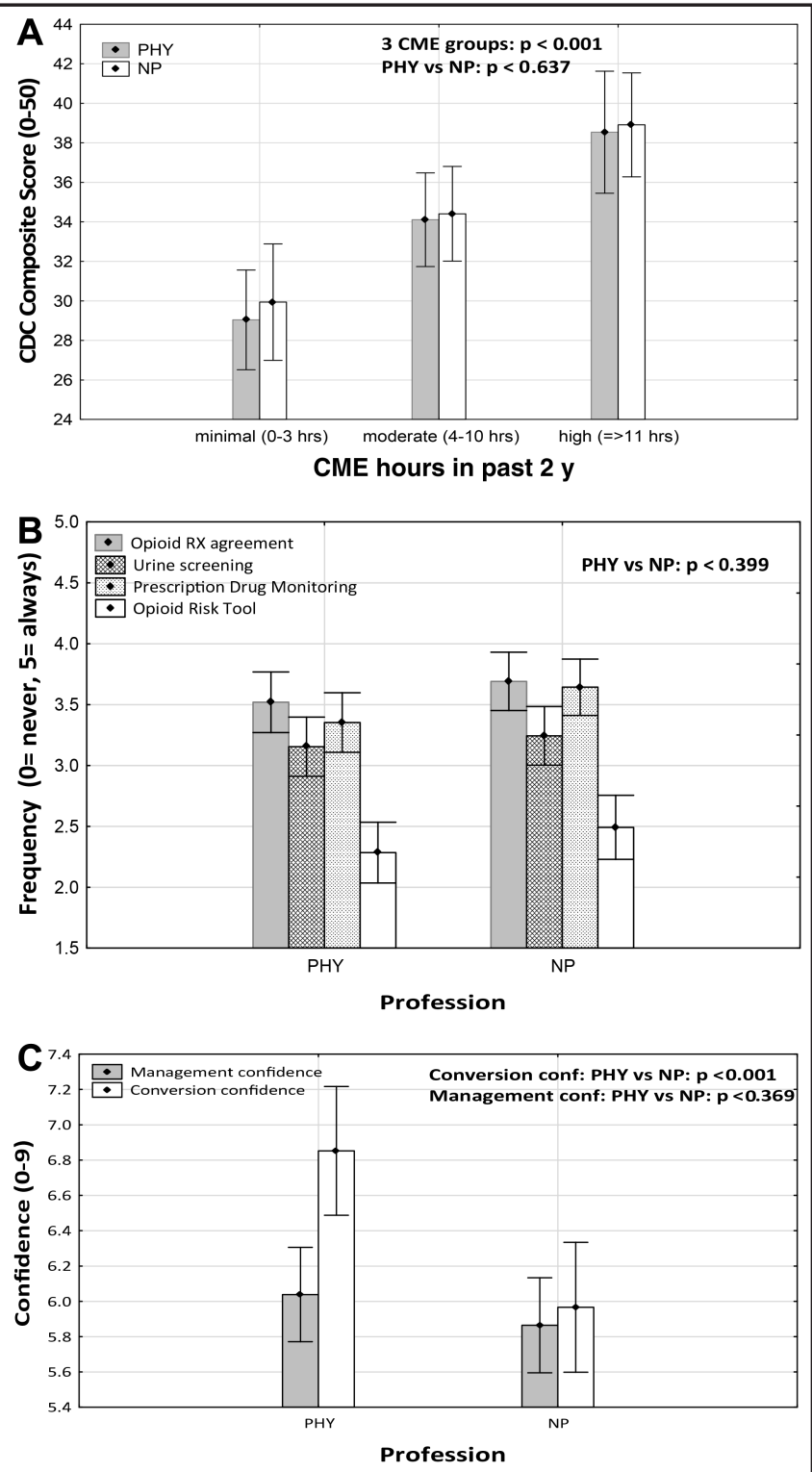

Figure 2. A, The physicians (PHY) and NPs do not differ in CDC Composite Score $(F=0.22 ; p<0.637)$ overall and at each level of CME hours. The three CME groups, regardless of profession, are significantly different from each other in the CDC $(F=20.98 ; p<0.001)$. B, The PHY and NP do not differ significantly overall $(F=1.02 ; p<0.399)$ or in any of the 4 specific CDC opioid management tools (all p, nonsignificant). C, PHY are more confident than NP in opioid dose conversion $(F=11.31 ; p<0.001)$ but equal in confidence in pain management $(F=0.81 ; p<0.369)$.

composite score of CDC Opioid Management Recommendations, the four specific practices, and confidence in pain management (all $p>0.05$ ). However, NPs were somewhat less confident in opioid dose conversion calculations (5.9 vs 6.9; $\mathrm{p}<0.001$, scale 0-9) compared to physicians. Despite this, the influence of CME was nearly identical for 
physician $(\mathrm{p}<0.001)$ and NP $(\mathrm{p}<0.001)$ participants in increasing adherence to CDC recommended practice and confidence ratings $(\mathrm{p}<0.001)$.

\section{Urban-rural comparison and CDC practices and provider confidence}

Figures 3A-3C show no significant differences between urban and rural providers' CDC Composite Score, four specific practices, or general confidence in CNCP management (all $\mathrm{p}>0.05$ ). CME influence on provider confidence ratings and adherence to recommended practices (not shown) were similar for both urban $(\mathrm{p}<0.001)$ and rural providers $(\mathrm{p}<0.001)$. However, compared to urban providers, rural providers expressed unique barriers given their geographic setting. Two barriers highlighted in this survey are rural providers' inadequate access to specialized pain clinics and to providers who prescribe medication assisted therapy (eg, buprenorphine) for opioid addiction or chronic pain management within 30 miles of their clinic $(\mathrm{p}<0.001)$.

\section{Provider CME preferences}

The means for participating providers' preferred formats for $\mathrm{CME}(0=$ not interested; $9=$ very interested) were conference lectures (5.3), self-guided modules on the Internet (5.3), and provider in-service trainings in their clinic (5.2). Training topics of greatest interest to Oregon providers were harm reduction in opioid prescribing (eg, naloxone, 5.4), medication assisted therapy (eg, buprenorphine, 5.3), and opioid tapering practices (5.1). Providers with fewer CME hours were significantly more interested in additional training $(\mathrm{p}<0.002)$. However, they did not differ from those with more CME hours $(p<0.724)$ in the type of training they desired.

\section{Pain specialists}

While only 17 pain specialist providers participated in this study, it is nevertheless instructive to see how they compared with the family practitioners and (other) medical specialist counterparts. For this comparison, we do not present a formal statistical comparison (eg, $F$ test) because of the unequal sample sizes and variances. Instead we present the means and 95\% confidence intervals for each of the three groups separately. As can be seen from Figures $4 \mathrm{~A}-4 \mathrm{C}$, the pain specialists had higher scores on the
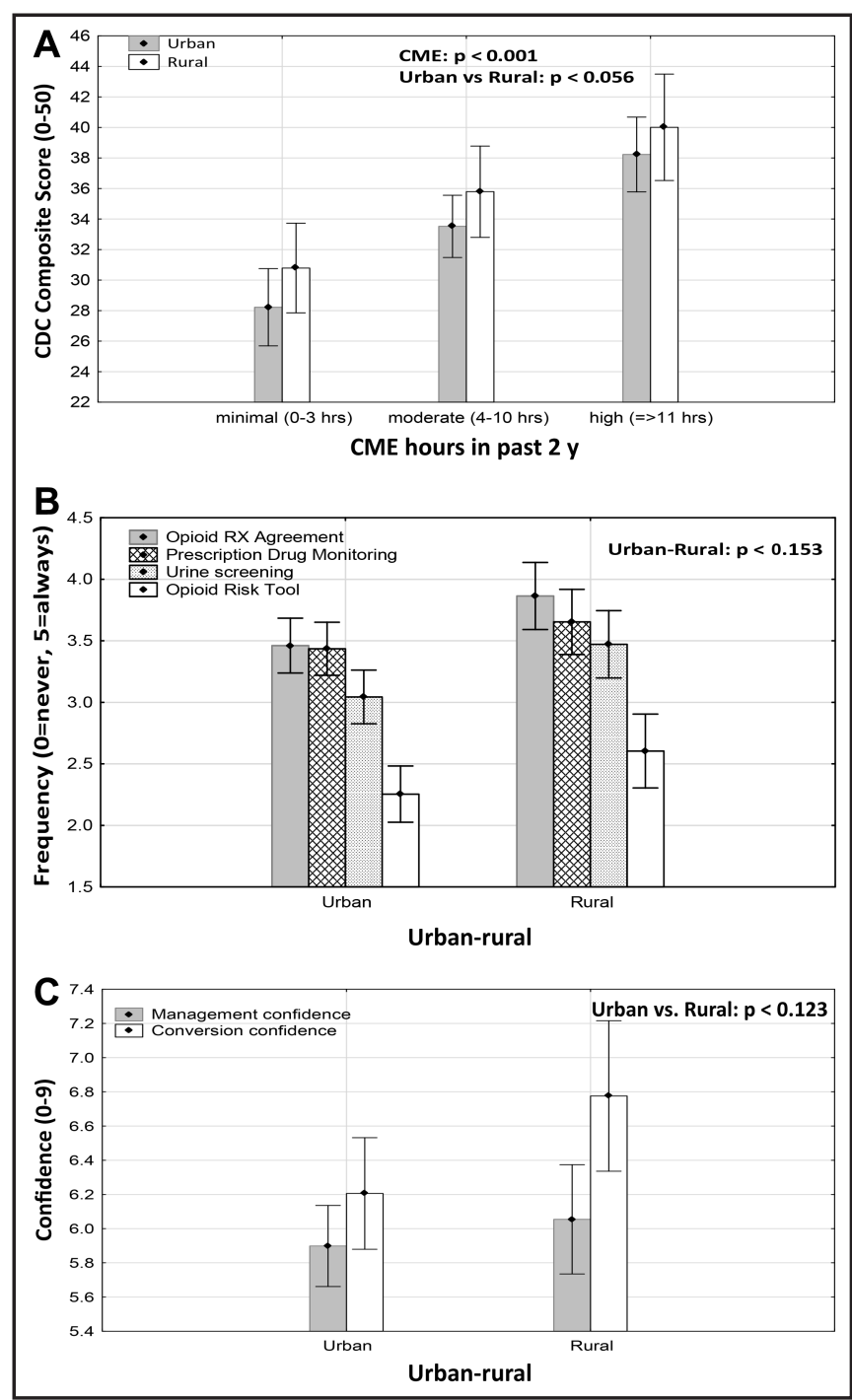

Figure 3. A, Compared to rural providers, urban providers show marginally higher scores overall $(F=3.66 ; p<0.056)$ but do not differ significantly at any one level of CME level (see 95\% CI). The three CME groups remain significant $(F=21.78 ; p<0.001) . B$, Urban and rural providers do not differ on any of the four specific $C D C$ opioid management tools $(p<0.399)$. C, Urban and rural providers do not differ in overall confidence $(F=2.11 ; p<0.123)$. Univariately, compared to urban providers, rural providers are more confident on conversion confidence $(F=4.18 ; p<0.042)$ but do not differ on management confidence $(F=0.59$; $\mathbf{p}<0.442)$.

CDC Composite Score (42.2 vs 37.6 and 23.7), confidence in pain management (8.4 vs 6.1 and 5.2 ), and conversion confidence ( 8.5 vs 6.8 and 5.0). The pain specialists also had considerably more CME hours (38.5 vs 10.1 and 7.3). Accordingly, they were least interested in additional training (1.5) compared to family providers (5.0) and other non-pain medical specialists (4.7, scale 0-9). These results provide some 

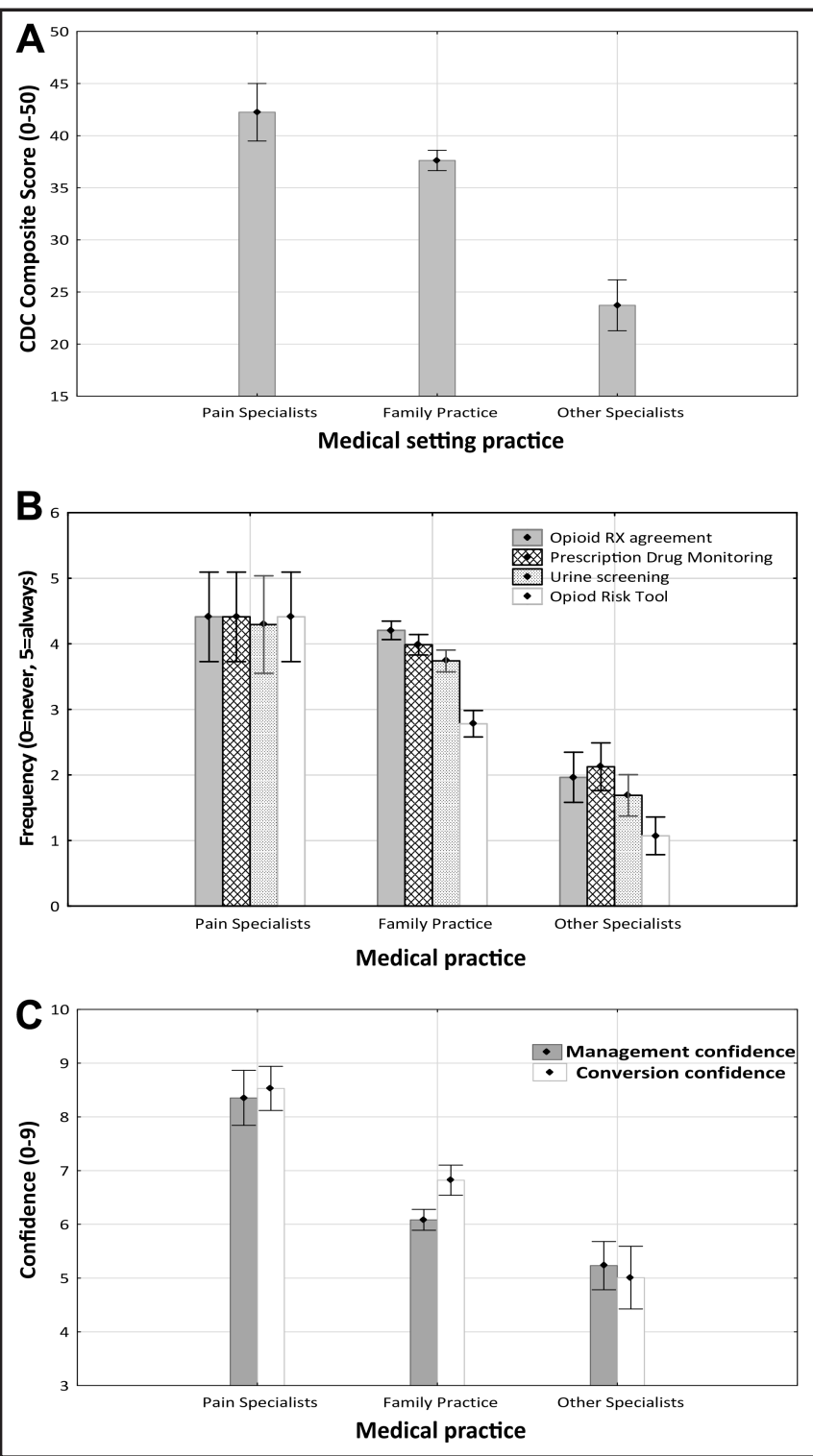

Figure 4. Pain specialists $(n=17)$ have higher CDC Composite Scores than family practice $(n=268)$ and other specialists $(n=112)$. No $F$ test comparison was conducted because of unequal Ns and variances. $B$, Pain specialists ( $n=17$ ) have higher frequency scores for the four specific CDC opioid management tools than family practice $(n=268)$ and other specialists $(n=112)$. No $F$ test comparison was conducted because of unequal Ns and variances. C, Pain specialists $(n=17)$ have higher Confidence Scores in both pain management and opioid dose conversion than family practice $(n=268)$ and other specialists $(n=112)$. No comparative $F$ test conducted because of unequal Ns and variances.

evidence for the face validity of the survey, and concurrent validity in that "known" groups responded in the way they were expected. Pain specialists were more proficient at utilizing the CDC guideline, thus providing some validity for the questions.

\section{DISCUSSION}

This evaluation of the 2016 Guideline for Prescribing Opioids for Chronic Pain, conducted only 6 months after its endorsement by the state of Oregon, addresses an important gap in the literature. Due to its recent release, there are no existing studies to our knowledge that examine provider perception and utilization of this guideline. The majority of the respondents was familiar with or had read the CDC guideline (91 percent). However, since only 57 percent of respondents report using this guideline in practice, increased efforts (eg, CME training) may be necessary to ensure the actual use of this guideline in clinical practice.

More than two thirds of the respondents (69 percent) were in support of the guideline's recommendation that clinicians avoid prescribing opioid doses greater than $90 \mathrm{MME} / \mathrm{d}$; thus highlighting the survey respondents' acceptance of this contested safety limit. Specific practices identified in the CDC guideline (eg, use of nonpharmacologic or nonopioid medications, use of an opioid risk tool, urine drug screening, PDMP review) provide tangible interventions that can be applied in the clinical setting. Utilization of these tools provides a backing for provider decision making and confidence by reducing the risk of adverse effects from opioid treatment.

This study analyzes the relationship between recent CME hours in CNCP and provider adherence to the 2016 CDC recommendations. The results are consistent with current literature that highlights the important role that CME plays in advancing provider knowledge and confidence in CNCP management and opioid prescribing but goes further to show that CME influences reported use of the guideline in addition to knowledge.

Previous provider surveys ${ }^{6,19-21}$ have identified CME as a viable means to increase provider knowledge and competency in managing CNCP. A pre-post survey of 45 resident physicians who underwent a focused educational training module on opioid prescribing for CNCP demonstrated increased knowledge and confidence in opioid prescribing. ${ }^{22}$ Similarly, a randomized pilot and feasibility trial with 81 general practitioners found that CME in CNCP increases provider knowledge of prescribing and decreases provider concerns related to prescribing. ${ }^{23}$ Not only are our results in line with these earlier findings that demonstrate that CME increases providers' knowledge or confidence, but 
also they show that providers' reported adherence to recommended practices is impacted by CME training.

More successful provider training has incorporated the implementation of practice-based interventions. Following the implementation of a multicomponent quality improvement intervention in three primary care practices in Pennsylvania, providers demonstrated an increase in knowledge, job-related satisfaction, and adherence to the opioid best practice guidelines including an increase in ordering urine drug screening. ${ }^{5}$ A survey of 710 Canadian family physicians identified provider access to a patient's opioid history from a PDMP, knowledge of risks and benefits of different opioids, access to pain specialist, and up to date guidelines on use of opioids in chronic pain as the most important enabling factors for optimizing use of opioids for CNCP. ${ }^{7}$ These findings are consistent with the National Academies of Science, Engineering \& Medicine's recent assertion that, "prescribing guidelines may be able to improve provider prescribing behavior, but may be most effective when accompanied by education and other means to facilitate intervention." $24(\mathrm{p} 10)$

Our data indicate that more CME hours in chronic pain care is positively associated with advancing provider knowledge, confidence, and adherence to current best practice recommendations in CNCP management and opioid prescribing. These findings were true among physicians and NPs as well as urban and rural providers. This study shows that an increase in CME dose from minimal to moderate and from moderate to high provides benefits. Provider preferences for CME format and content reported here should be considered in the future design and implementation of CNCP-related CME.

This is the first survey to compare physicians and NPs adherence to nationally recommended practices in CNCP management. Overall, our findings show the similarities between physician and NPs' confidence in managing chronic pain, but NPs were somewhat less confident in MME conversions. Current literature emphasizes the important role of physicians in pain care, yet fails to highlight the work of NPs. A chart review of NP management of 50 CNCP patients at 175 percent of the poverty level or less showed that NPs consistently used practices advocated by the 2016 CDC guideline. ${ }^{25}$ A follow-up study on patient perception of NP management of chronic pain is needed.
This study also explored differences in rural and urban providers' management of CNCP. It was seen that rural providers in Oregon were marginally more confident than urban providers in treating CNCP patients. Furthermore, there were no significant differences in these groups' use of CDC recommended practices. Unique challenges identified by rural respondents were consistent with themes in the current literature: provider retention, ${ }^{26,27}$ disparities in infrastructure and professional capacity, ${ }^{28}$ and inadequate access to pain specialists and other healthcare services. ${ }^{29}$

This survey has limitations. Although the number of respondents surpassed the original target of 350 providers, nearly one fifth of respondents had incomplete responses. This limitation may be attributed to survey length, competing demands on provider time, and provider willingness to complete the survey. The average time to complete the survey was 20 minutes. Also we did not adequately assess for the patient case mix or examine how this might have influenced our results. Moreover, it is not known if the individuals who completed our survey were more confident and more adherent to CDC best pain practices compared to those who did not. Future studies are needed to determine if reported practices in pain management and opioid prescribing translate to actual practices. Additional work is also needed to examine specific guideline recommendations by pain experts, most notably what should be done with those patients who are currently on opioid dosages over $90 \mathrm{MME} / \mathrm{d}$.

\section{CONCLUSIONS}

Like many states, Oregon voted to endorse the 2016 CDC guideline as their foundation for opioid prescribing. ${ }^{30}$ This is the first statewide study to evaluate provider utilization of the 2016 CDC opioid prescribing recommendations, and the results support standardized CME requirements in chronic pain management for all practicing healthcare providers. Opioid prescribing decision-making tools and their widespread usage should be evaluated as a potential risk mitigation strategy in the current opioid epidemic. In this respect, expanded efforts are needed to increase provider familiarity with and use of the 2016 CDC guideline, while balancing the recommendations with the responsibility to meet the individualized needs of each patient. 
Jean C. McCalmont, DNP, FNP, Nursing, Oregon Health $\&$ Science University, Portland, Oregon.

Kim D. Jones, PhD, FNP, FAAN, Nursing, Oregon Health \& Science University, Portland, Oregon.

Robert M. Bennett, MD, FRCP, MACR, Nursing, Oregon Health \& Science University, Portland, Oregon.

Ronald Friend, PhD, Psychology, Stony Brook University, Stony Brook, New York.

\section{REFERENCES}

1. Gureje O, Von Korff M, Simon GE, et al.: Persistent pain and well-being: A World Health Organization study in primary care. JAMA. 1998; 280(2): 147-151.

2. Rasu RS, Sohraby R, Cunningham L, et al.: Assessing chronic pain treatment practices and evaluating adherence to chronic pain clinical guidelines in outpatient practices in the United States. J Pain. 2013; 14(6): 568-578.

3. Jamison RN, Scanlan E, Matthews ML, et al.: Attitudes of primary care practitioners in managing chronic pain patients prescribed opioids for pain: A prospective longitudinal controlled trial. Pain Med. 2014; 17: 99-113.

4. Mezei L, Murinson BB: Pain education in North American medical schools. J Pain. 2011; 12(12): 1199-1208.

5. Canada RE, DiRocco D, Day S: A better approach to opioid prescribing in primary care. J Family Pract. 2014; 63(6): E1-E8.

6. Keller CE, Ashrafioun L, Neumann AM, et al.: Practices, perceptions, and concerns of primary care physicians about opioid dependence associated with the treatment of chronic pain. Subst Abuse. 2012; 33(2): 103-113.

7. Allen MJ, Asbridge MM, Macdougall PC, et al.: Self-reported practices in opioid management of chronic noncancer pain: A survey of Canadian family physicians. Pain Res Manag. 2013; 18(4): 177-184.

8. Potter M, Schafer S, Gonzalez-Mendez E, et al.: Opioids for chronic nonmalignant pain. Attitudes and practices of primary care physicians in the UCSF/Stanford Collaborative Research Network. University of California, San Francisco. J Fam Pract. 2001; 50(2): 145-151.

9. Green CR, Wheeler J, LaPorte F, et al.: How well is chronic pain managed? Who does it well? Pain Med. 2002; 3: 56-65.

10. Centers for Disease Control and Prevention: CDC Guideline for Prescribing Opioids for Chronic Pain-United States, 2016. Available at http://www.cdc.gov/mmwr/volumes/65/rr/rr6501e1. htm. Published March 18, 2016. Accessed September 2, 2016.

11. Pergolizzi JV, Raffa RB, LeQuang JA: The Centers for Disease Control and Prevention opioid guidelines: Potential for unintended consequences and will they be abused? J Clin Pharm Ther. 2016; 41:592-593.

12. Bero LA, Grilli R, Grimshaw JM, et al.: Getting research findings into practice: Closing the gap between research and practice: An overview of systematic reviews of interventions to promote the implementation of research findings. BMJ. 1998; 317(7156): 465-468.

13. Fudin J, Raouf M, Wegrzyn EL: Opioid dosing policy: Pharmacological considerations regarding equianalgesic dosing. Available at http://c.ymcdn.com/sites/www.integrativepain management.org/resource/resmgr/docs/MEDD_White_Paper. pdf. Accessed October 8, 2017.
14. Hollingshead NA, Meints S, Middleton SK, et al.: Examining influential factors in providers' chronic pain treatment decisions: A comparison of physicians and medical students. BMC Med Educ. 2015; 15(164): 1-8.

15. Hutchinson K, Moreland A, de Williams A, et al.: Exploring beliefs and practice of opioids prescribing for persistent noncancer pain by general practitioners. Eur J Pain. 2007; 11: 93-98. 16. Schneider JP: CDC recommendations fall short and ignore important aspects of pain management. Pract Pain Manage. 2016; 16(3): 18-21.

17. Morse JS, Stockbridge H, Egan KB, et al.: Primary care survey of the value and effectiveness of the Washington State Opioid Dosing Guideline. J Opioid Manag. 2011; 7(6): 427-433.

18. Oregon.gov: Continuing education. Available at https:// www.oregon.gov/omb/Topics-of-Interest/Pages/ContinuingEducation.aspx. Accessed April 4, 2017.

19. Hooten WM, Bruce BK: Beliefs and attitudes about prescribing opioids among healthcare providers seeking continuing medical education. J Opioid Manag. 2011; 7(6): 417-424.

20. Lalonde L, Leroux-Lapointe V, Choiniere, M, et al: Knowledge, attitudes and beliefs about chronic noncancer pain in primary care: A Canadian survey of physicians and pharmacists. Pain ResManag. 2014; 19(5): 241-250.

21. Kavukcu E, Akdeniz M, Huseyin H, et al.: Chronic non-cancer pain management in primary care: family medicine physicians' risk assessment of opioid misuse. Postgrad Med. 2015; 127(1): 22-26.

22. Regunath $\mathrm{H}$, Cochran $\mathrm{K}$, Cornell $\mathrm{K}$, et al.: Is it painful to manage chronic pain? A cross-sectional study of physicians in-training in a university program. Missouri Med. 2016; 113(1): 72-78.

23. McCracken LM, Velleman SC, Eccleston C: Patterns of prescription and concerns about opioid analgesics for chronic non-malignant pain in general practice. Primary Health Care Res Dev. 2012; 9: 146-156.

24. Committee on Pain Management and Regulatory Strategies to Address Prescription Opioid Abuse: Pain Management and the Opioid Epidemic. Washington, DC: The National Academies Press, 2017: 1-14.

25. Creech CJ, Clark S, Grube G, et al.: Nurse practitioner management of chronic musculoskeletal pain: A chart review. Nurse Pract. 2011; 36(9): 29-36.

26. National Center for Health Statistics: Healthy people 2010 final review. Available at https://www.cdc.gov/nchs/data/hpdata2010/ hp2010_final_review.pdf. Accessed September 7, 2016.

27. Council of State Governments: Health care workforce shortages critical in rural America, CAPITOL facts \& figures. Available at $h t t p: / /$ knowledgecenter.csg.org/kc/system/files/Health_Care_Workforce_ Shortages_Critical_in_Rural_America.pdf. Accessed April 2, 2017.

28. Gamm L, Hutchison, L, Bellamy G, et al.: Rural healthy people 2010: Identifying rural health priorities and models for practice. J Rural Health. 2002; 18(1): 9-14.

29. Remster EN, Marx TL: Barriers to managing chronic pain: A pilot of prescriber perceptions in rural Appalachia. J Pain Symptom Manag. 2008; 36(3): e1-e2.

30. Oregon Health Authority: Oregon opioid prescribing guidelines: Recommendations for the safe use of opioid medications. Available at http://www.oregon.gov/obnm/rules/opioidprescrib ingguidelines.pdf. Accessed February 16, 2017.

31. Centers for Disease Control and Prevention: CDC Guideline for Prescribing Opioids for Chronic Pain. Available at https:// www.cdc.gov/drugoverdose/pdf/guidelines_at-a-glance-a.pdf. Accessed October 8, 2017. 


\section{Appendix 1: Survey questions}

1. Do you provide care to patients living with chronic non-cancer pain (pain $>3$ months)?

a. Yes

b. No $\rightarrow$ Will skip ahead to question $\# 12$.

2. How confident are you managing chronic non-cancer pain?

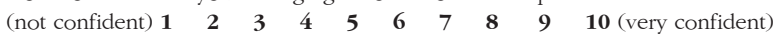

3. Approximately what percentage of your patient panel is receiving opioids for an extended period of time ( $>3$ months) for the management of chronic non-cancer pain? a. $0 \%$

b. $1-10 \%$

c. $11-20 \%$

d. $21-30 \%$

e. $31-40 \%$

f. $41-50 \%$

g. $>50 \%$

4. Approximately what percentage of your patients did you start on opioids?

a. $0 \%$

b. $1-10 \%$

c. $11-20 \%$

d. $21-30 \%$

e. $31-40 \%$

f. $41-50 \%$

g. $>50 \%$

5. How concerned are you that your patients on long-term opioids will..

a. Develop psychological dependence?

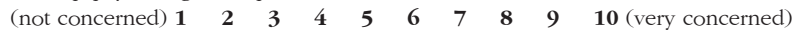

b. Develop physical dependence (experience withdrawal symptoms if medication is tapered too quickly or stopped abruptly)?

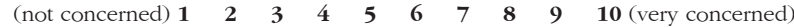

c. Develop opioid use disorder (addiction)?

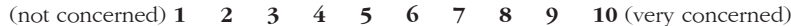

d. Divert their opioids?

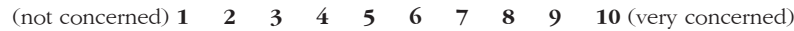

e. Overdose?

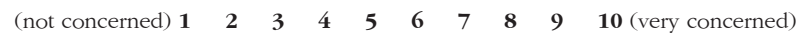

f. Develop opioid-induced hyperalgesia?

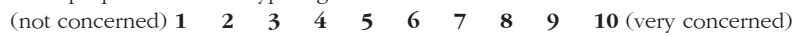

6. When seeing patients with chronic non-cancer pain, how often do you or your rooming assistant (MA, RN) document the following characteristics at the initial visit?

a. Pain duration (acute vs. chronic)

Never, Almost never, Sometimes, Often, Almost Always, Always

b. Pain location

Never, Almost never, Sometimes, Often, Almost Always, Always

c. Pain condition (e.g. low back pain, fibromyalgia, diabetic neuropathy)

Never, Almost never, Sometimes, Often, Almost Always, Always

d. Pain severity score (e.g. 0-10 pain scale)

Never, Almost never, Sometimes, Often, Almost Always, Always

e. Level of function

Never, Almost never, Sometimes, Often, Almost Always, Always

7. When seeing patients with chronic non-cancer pain, how often do you or your rooming assistant (MA, RN) document the following characteristics at every follow up visit? a. Pain duration (acute vs. chronic)

Never, Almost never, Sometimes, Often, Almost Always, Always

b. Pain location

Never, Almost never, Sometimes, Often, Almost Always, Always

c. Pain condition (e.g. low back pain, fibromyalgia, diabetic neuropathy)

Never, Almost never, Sometimes, Often, Almost Always, Always

d. Pain severity score (e.g. 0-10 pain scale)

Never, Almost never, Sometimes, Often, Almost Always, Always

e. Level of function

Never, Almost never, Sometimes, Often, Almost Always, Always

8. Before prescribing opioids for a patient with chronic non-cancer pain, how often do you employ the following practices?

a. Select non-pharmacologic therapy and non-opioid medications instead of opioids

Not applicable, Never, Almost never, Sometimes, Often, Almost Always, Always

b. Screen for comorbid mental health disorder

Not applicable, Never, Almost never, Sometimes, Often, Almost Always, Always

c. Use an opioid risk assessment tool (e.g. Opioid Risk Tool)

Not applicable, Never, Almost never, Sometimes, Often, Almost Always, Always

d. Review patient's personal or family history of substance abuse

Not applicable, Never, Almost never, Sometimes, Often, Almost Always, Always

e. Require an opioid treatment agreement

Not applicable, Never, Almost never, Sometimes, Often, Almost Always, Always

$\mathrm{f}$. Establish treatment goals with the patient

Not applicable, Never, Almost never, Sometimes, Often, Almost Always, Always 
g. Conduct urine drug screening

Not applicable, Never, Almost never, Sometimes, Often, Almost Always, Always

h. Access the Oregon Prescription Drug Monitoring Program

Not applicable, Never, Almost never, Sometimes, Often, Almost Always, Always

i. Refer patient to pain management specialist

Not applicable, Never, Almost never, Sometimes, Often, Almost Always, Always

j. Assess for benzodiazepine use

Not applicable, Never, Almost never, Sometimes, Often, Almost Always, Always

9. In patients with chronic non-cancer pain who are continuing on opioids, how often do you employ the following practices at least yearly?

a. Continue to encourage non-pharmacologic therapy

Not applicable, Never, Almost never, Sometimes, Often, Almost Always, Always

b. Review patient's personal or family history of substance abuse

Not applicable, Never, Almost never, Sometimes, Often, Almost Always, Always

c. Review and update opioid treatment agreement

Not applicable, Never, Almost never, Sometimes, Often, Almost Always, Always

d. Reassess treatment goals

Not applicable, Never, Almost never, Sometimes, Often, Almost Always, Always

e. Conduct urine drug screening

Not applicable, Never, Almost never, Sometimes, Often, Almost Always, Always

f. Access the Oregon Prescription Drug Monitoring Program

Not applicable, Never, Almost never, Sometimes, Often, Almost Always, Always

g. Refer patient to pain management specialist (if not previously referred)

Not applicable, Never, Almost never, Sometimes, Often, Almost Always, Always

h. Offer narcan when factors that increase risk for opioid-related harm are present

Not applicable, Never, Almost never, Sometimes, Often, Almost Always, Always

10. What resources do you utilize when developing a treatment plan for patients with chronic non-cancer pain? (Select all that apply.)

a. Online evidence-based, peer-reviewed resource (e.g. UpToDate)

b. Online medication reference tool (e.g. Epocrates)

c. Clinical practice guideline

d. Primary care colleagues

e. Specialty care colleagues

f. Clinical pharmacist

g. Telehealth services

h. I do not routinely develop treatment plans for patients with chronic non-cancer pain

i. None of the above

11. If you utilize a clinical practice guideline when developing a patient treatment plan, please indicate which guideline you use. (Select all that apply.)

a. Oregon Pain Guidance, Southern Oregon Opioid Prescribing Guidelines

b. Washington State Agency Medical Directors Group, Interagency Guideline on Prescribing Opioids for Pain

c. American Pain Society, Guideline for the Use of Chronic Opioid Therapy in Chronic Non-cancer Pain

d. Veteran's Health Administration/Department of Defense, Clinical Practice Guideline for Management of Opioid Therapy for Chronic Pain

e. Centers for Disease Control, Guideline for Prescribing Opioids for Chronic Pain

f. Organization-specific guideline

g. I do not use a clinical practice guideline.

h. Other

12. How familiar are you with the Center for Disease Control's Guidelines for Prescribing Opioids for Chronic Pain that were released in March 2016?

a. Not familiar with the guideline

b. Familiar, but have not read

c. Have read, but not applied in practice

d. Have read and applied in practice

13. The 2016 CDC's Guidelines for Prescribing Opioids for Chronic Pain recommends that clinicians avoid prescribing opioid doses equal to or greater to 90 morphine milligram equivalents/day (equivalent to $60 \mathrm{mg}$ oxycodone/day). Based on your experience, this threshold dose recommendation is:

a. Too high

b. Reasonable

c. Too low

14. How confident are you calculating opioid conversion doses (morphine equivalents) of commonly used opioids?

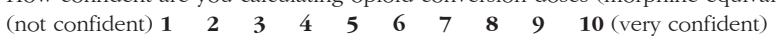

15. There is adequate access to providers who prescribe medication-assisted treatment (e.g. buprenorphine) for opioid addiction or chronic pain management within 30 miles of my clinic:

Strongly agree, Agree, Somewhat agree, Somewhat disagree, Disagree, Strongly disagree

16. There is adequate access to a specialized pain clinic within 30 miles of my clinic:

Strongly agree, Agree, Somewhat agree, Somewhat disagree, Disagree, Strongly disagree

17. How problematic are the following issues in managing chronic non-cancer pain patients?

a. Insufficient time

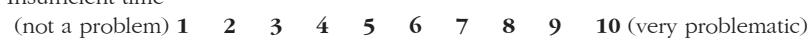

b. Inadequate improvement with non-opioid medications

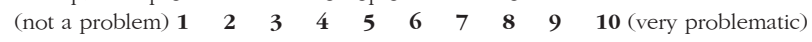

c. Inadequate improvement on opioids

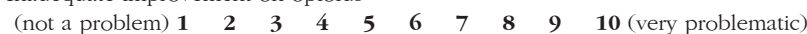


d. Inadequate access to non-pharmacologic therapies (e.g. physical therapy, cognitive behavioral therapy)

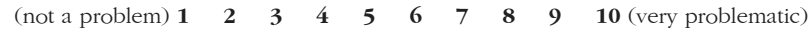

e. Inadequate improvement with non-pharmacologic therapies

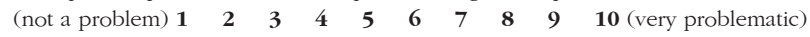

f. Inadequate access to complementary and alternative medicine therapies (e.g. massage, acupuncture)

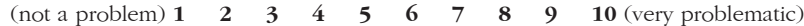

g. Inadequate improvement with complementary and alternative medicine therapies

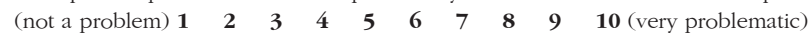

h. Inadequate access to a pain specialist or specialized pain clinic for patients on the Oregon Health Plan (OHP)

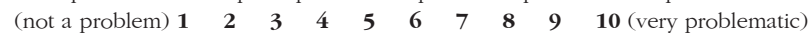

i. Complex patients with multiple comorbidities

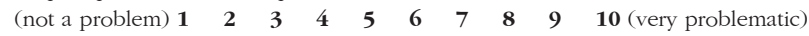

j. Patient unwillingness to engage in care or utilize non-opioid therapies

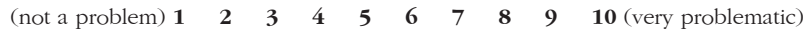

k. Patients' inability to pay for treatment/services not covered by insurance

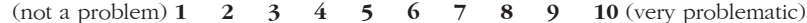

18. What services does your clinic/organization currently offer? (Select all that apply.)

a. Clinical pharmacist on staff

b. Behavioral health specialist on staff

c. Medication-assisted treatment (e.g. buprenorphine) prescriber on staff

d. Physical therapist on staff

e. Acupuncturist on staff

f. Chronic pain group visits

g. None of the above.

19. How much additional training would you find helpful in managing chronic non-cancer pain?

a. Assessment of chronic non-cancer pain

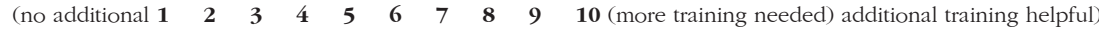

b. Treatment of chronic non-cancer pain

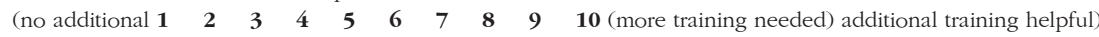

c. Non-opioid and non-pharmaceutical treatment options

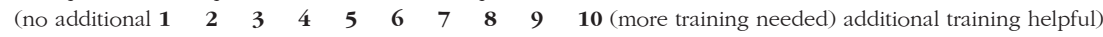

d. Opioid prescribing best practices

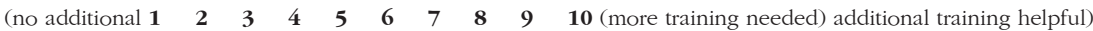

e. Opioid tapering best practices

(no additional $\begin{array}{llllllllll}1 & 2 & 3 & 4 & 5 & 6 & 7 & 8 & 9 & 10\end{array}$ (more training needed) additional training helpful)

f. Medication-assisted treatment (e.g. buprenorphine)

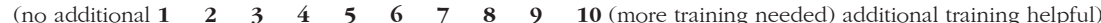

g. Harm reduction in opioid prescribing (e.g. narcan)

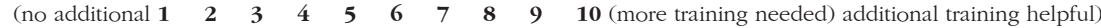

h. Access to the Oregon Prescription Drug Monitoring Program

(no additional $\begin{array}{llllllllll}1 & 2 & 3 & 4 & 5 & 6 & 7 & 8 & 9 & 10\end{array}$ (more training needed) additional training helpful)

i. Patient engagement techniques (e.g. motivational interviewing, mindfulness, meditation)

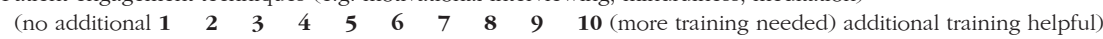

20. How interested are you in the following ways to learn more about opioid prescribing best practices?

a. Formal lecture-attend a conference

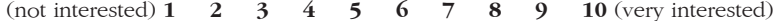

b. Formal lecture-provider in-service training at my clinic

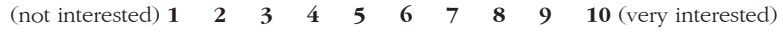

c. Formal lecture-attend a community training

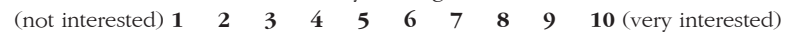

d. Interactive activities or courses-attend a conference

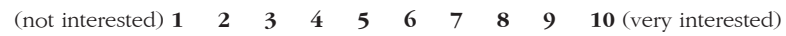

e. Interactive activities or courses-provider in-service training at my clinic

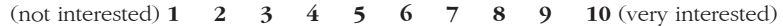

f. Interactive activities or courses - attend a community training

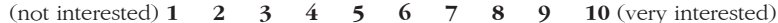

g. Self-learning modules-paper format

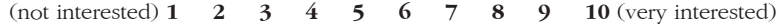

h. Self-learning modules-Internet based

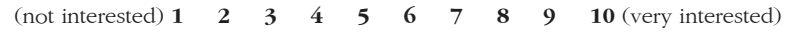

i. Self-learning modules-podcast

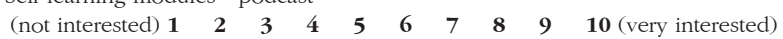

21. How many hours of formal continuing education on chronic non-cancer pain management have you had in the past two years? ( 0 -100)

22. How many hours of formal continuing education on opioid prescribing have you had in the past two years? $(0-100)$

23. What is your profession?

a. Physician

b. Physician Assistant

c. Nurse Practitioner

d. Medical Resident

e. Other (please specify): 
24. How many years have you been in practice? (Drop down $<1,1,2 \ldots 60,>60$ )

25. What is your age? Drop down (20-90)

26. What is your gender?
a. Male
b. Female
c. Transgender
d. Prefer not to answer
e. Other (please specify)

27. Which best represents your ethnicity?

a. Hispanic

b. Non- Hispanic

28. Which best represents your race?

a. American Indian or Alaska Native

b. Asian

c. Black or African American

d. Native Hawaiian or Other Pacific Islander

e. White

29. Which best describes your primary place of employment?

a. Urban/suburban

b. Rural

c. Frontier

30. Which region best describes the location of your primary place of employment?

a. Portland Metro area

b. Southern Oregon

c. Central Oregon

d. Eastern Oregon

c. Willamette Valley

f. Oregon Coast

g. Mt. Hood/Columbia River Gorge

h. My practice is not in Oregon

31. What is your practice setting?
a. Family Medicine
b. General Internal Medicine
c. Psychiatric/Mental Health
d. Pain management clinic
e. Rheumatology
f. Neurology
g. Orthopedics
h. Anesthesiology
i. Other (please specify):

\section{Appendix 2: Synopsis of CDC's Guideline for Prescribing Opioids for Chronic Pain ${ }^{31}$}

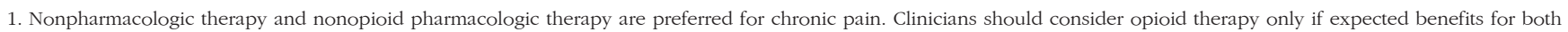
pain and function are anticipated to outweigh risks to the patient. If opioids are used, they should be combined with nonpharmacologic therapy and nonopioid pharmacologic therapy, as appropriate.

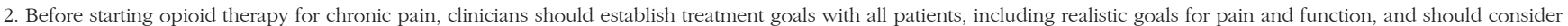
how opioid therapy will be discontinued if benefits do not outweigh risks. Clinicians should continue opioid therapy only if there is clinically meaningful improvement in pain and function that outweighs risks to patient safety.

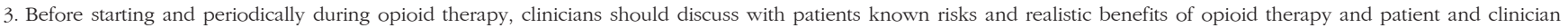
responsibilities for managing therapy.

4. When starting opioid therapy for chronic pain, clinicians should prescribe immediate-release opioids instead of extended-release/long-acting (ER/LA) opioids.

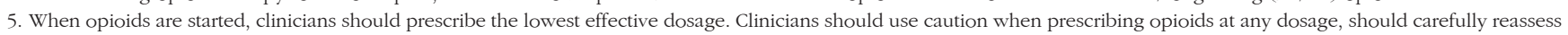
evidence of individual benefits and risks when considering increasing dosage to $\geq 50 \mathrm{MME} / \mathrm{d}$, and should avoid increasing dosage to $\geq 90 \mathrm{MME} / \mathrm{d}$ or carefully justify a decision to titrate dosage to $\geq 90 \mathrm{MME} / \mathrm{d}$.

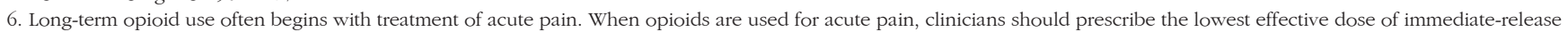
opioids and should prescribe no greater quantity than needed for the expected duration of pain severe enough to require opioids. Three days or less will often be sufficient; more than seven days will rarely be needed.

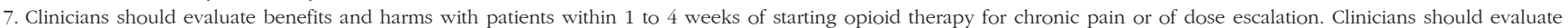
benefits and harms of continued therapy with patients every 3 months or more frequently. If benefits do not outweigh harms of continued opioid therapy, clinicians should optimize other therapies and work with patients to taper opioids to lower dosages or to taper and discontinue opioids.

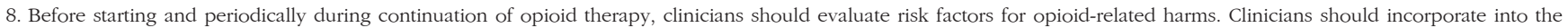
management plan strategies to mitigate risk, including considering offering naloxone when factors that increase risk for opioid overdose, such as history of overdose, history of substance use disorder, higher opioid dosages ( $\geq 50 \mathrm{MME} / \mathrm{d}$ ), or concurrent benzodiazepine use, are present.

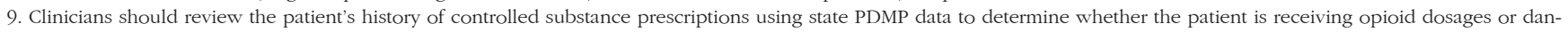
gerous combinations that put him or her at high risk for overdose. Clinicians should review PDMP data when starting opioid therapy for chronic pain and periodically during opioid therapy for chronic pain, ranging from every prescription to every 3 months.

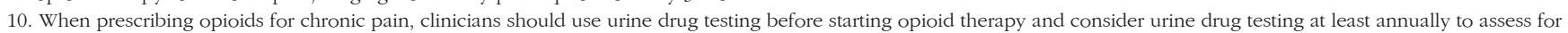
prescribed medications as well as other controlled prescription drugs and illicit drugs.

11. Clinicians should avoid prescribing opioid pain medication and benzodiazepines concurrently whenever possible.

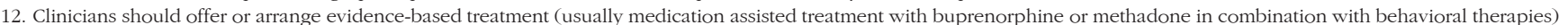
for patients with opioid use disorder. 